\title{
Dermatomyositis associated with gastrointestinal malignancies - report of two cases and a literature review
}

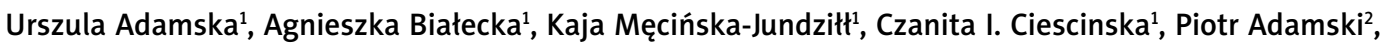 \\ Rafał Czajkowski ${ }^{1}$
}

${ }^{1}$ Chair of Dermatology, Sexually Transmitted Diseases, and Immunodermatology, Faculty of Medicine in Bydgoszcz, Nicolaus Copernicus University in Torun, Poland

${ }^{2}$ Department of Cardiology and Internal Medicine, Collegium Medicum, Nicolaus Copernicus University, Bydgoszcz, Poland

Adv Dermatol Allergol 2021; XXXVIII (2): 343-345

DOI: https://doi.org/10.5114/ada.2021.106217

Dermatomyositis is an idiopathic inflammatory myopathy with specific cutaneous lesions that are probably due to an autoimmune reaction. The exact pathogenesis is not fully understood, but drugs, infectious agents, or underlying malignancies may be the cause of dermatomyositis. The first report of dermatomyositis related to malignancy was published in 1916 [1]. Since that time the association between dermatomyositis and cancer has been extensively studied. It is difficult to determine whether malignancies contribute to the development of dermatomyositis or whether dermatomyositis contributes to the development of cancer. Some studies have reported a 3-to 6-fold higher risk of malignancy in patients with dermatomyositis [2]. Skin and muscle symptoms may precede the clinical diagnosis of cancer, appear simultaneously, or only after the detection of the tumour. However, they most frequently occur during the first year after the initial cancer diagnosis [3, 4].
We present 2 cases of dermatomyositis, diagnosed according to the Bohan and Peter criteria, that were associated with cancer and developed shortly after diagnosis.

A 73-year-old female patient with a history of hypertension and hypercholesterolaemia presented with extensive skin lesions and weakness of the upper body part muscles for 1 month. There was no known family history of skin diseases, and no history of infection, trauma, drug use, or toxic exposure. Physical examination revealed redness of the face, neck and shoulders, Gottron's papules over the joints of the hands, and ecchymosis and telangiectasias in the nail shafts of the fingers (Figures $1 \mathrm{~A}, \mathrm{~B}$ ). Complete blood count, urinalysis, and other routine laboratory tests returned normal, except elevated serum transaminases: alanine aminotransferase (ALT) $60 \mathrm{U} / \mathrm{l}$ (reference range: $10-49 \mathrm{U} / \mathrm{l}$ ), aspartate aminotransferase (AST) $116 \mathrm{U} / \mathrm{l}$ (reference range: 8-34 U/l), creatinine phosphokinase (CK) $2062 \mathrm{U} / \mathrm{l}$ (reference range: 29-168 U/I),
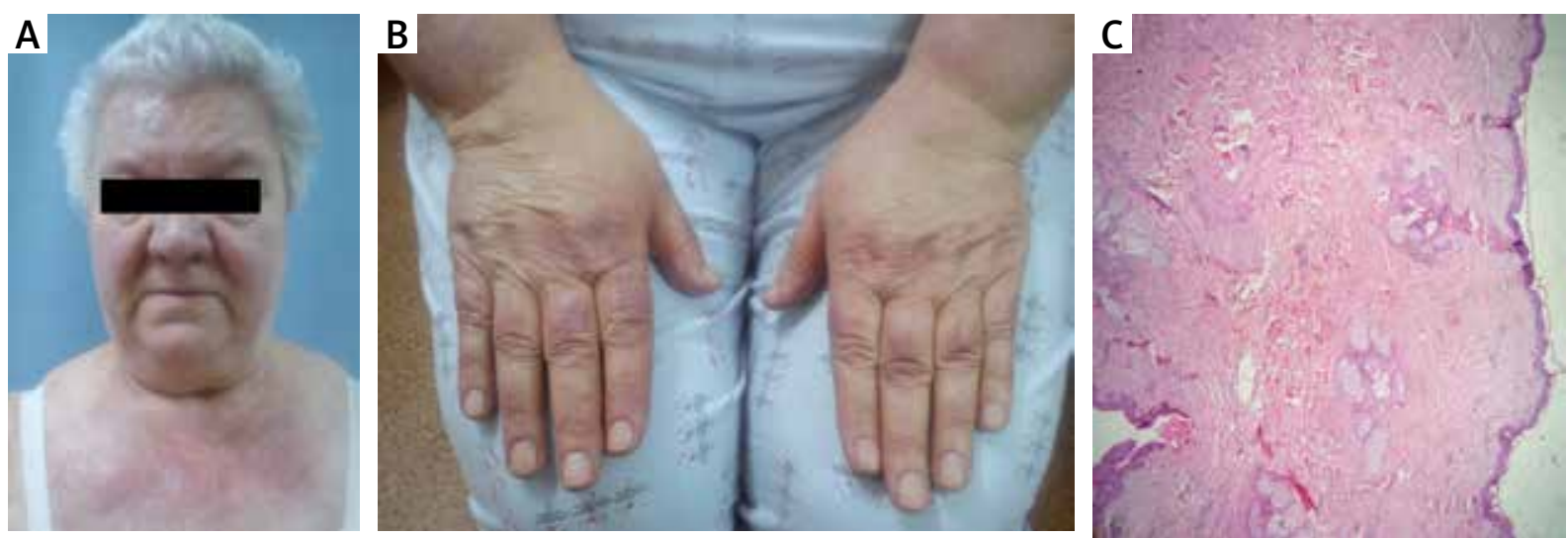

Figure 1. A reddish periorbital oedema and erythema of the face and the neck V-region (A), ecchymosis and telangiectasia of the nail shafts of the fingers with Gottron's papules over both metacarpophalangeal and interphalangeal regions of the hand (B). A muscle biopsy with nonspecific inflammatory changes (H\&E staining, $\times 100)$ (C)

Address for correspondence: Urszula Adamska MD, Chair of Dermatology, Sexually Transmitted Diseases, and Immunodermatology, Faculty of Medicine, Nicolaus Copernicus University, Bydgoszcz, Poland, phone: +48 505453743, e-mail: urszula.randzio@gmail.com Received: 23.09.2019, accepted: 30.09.2019. 

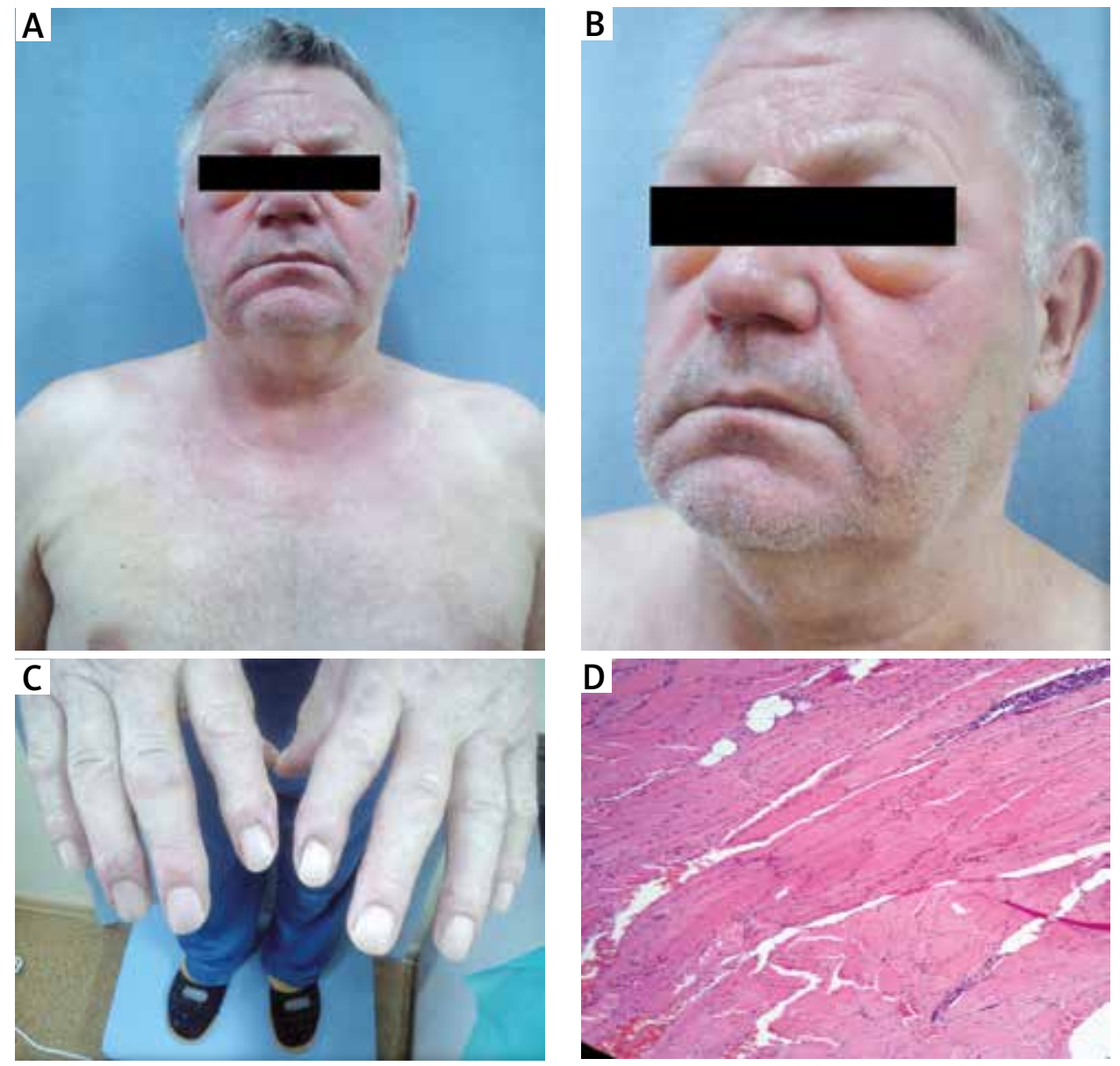

Figure 2. Severe periorbital oedema, violaceous erythema over the front of the chest $(\mathrm{V}$ sign) $(\mathrm{A}, \mathrm{B})$ and Gottron's papules of the finger joints (C). Histopathology of the deltoid muscle biopsy taken from the right arm showed a small amount of necrotic and regenerating fibres (H\&E staining, $100 \times$ ) (D)

and lactate dehydrogenase $(\mathrm{LDH}) 388 \mathrm{U} / \mathrm{l}$ (reference range: 120-246 U/I). Autoantibody screening revealed positive antinuclear antibodies (ANA) with a titre $1: 320$ of homogenous pattern, and no specific ANA was found. The skin and muscle biopsy showed nonspecific inflammatory changes (Figure 2 C), while electromyography (EMG) revealed myogenic muscle damage. A diagnosis of dermatomyositis based on the clinical and laboratory features was made; glucocorticoids treatment was started (prednisolone $0.6 \mathrm{mg} / \mathrm{kg} /$ day), and gradual improvement was observed. A number of additional tests were performed to determine the cause of the disease, and only the stool occult blood test was positive, whereas the other tests were not clinically relevant. Therefore, extensive evaluation of cancer-associated symptoms was performed. The patient was referred to an oncology centre where she was diagnosed with colorectal cancer and eventually died due to complications after tumour removal surgery.

A 72-year-old man was admitted to the hospital due to intensifying ocular swelling and redness accompanied by increasing fatigue over the previous 2 weeks. The symptoms had initially been considered allergic and were treated with low doses of glucocorticoids and antihistamines with no improvement. Despite tizanidine and diclofenac treatment, weakness of the shoulder girdle muscles was progressing continuously. The patient had a history of hypertension and impaired glucose tolerance, and his chronic medications included perindopril and amlodipine. Upon examination the patient presented very intensified reddish periorbital oedema and erythema of the face, neck, and back. Erythematous, scaly eruption, small ulcerations, and telangiectasia over both metacarpophalangeal and interphalangeal regions of the dorsal aspect of the hand were also observed (Figures $2 \mathrm{~A}-\mathrm{C})$. Laboratory tests revealed elevated serum glucose, ALT 82 U/I, AST 108 U/I, CK 2183U/l, and LDH 329 U/l. ANA was positive with a titre of $1: 640$, and autoantibodies against transcription intermediary factor $1 \gamma$ (anti-TIF-1 $\gamma$ antibody) were detected in immunoblotting assay. EMG of both shoulder and tibial muscles revealed myogenic muscle damage. A histological examination of specimens obtained from the right deltoid muscle revealed a small amount of necrotic and regenerating fibres (Figure $2 \mathrm{D}$ ). Dermatomyo- 
sitis was diagnosed based on the typical physical findings, including the presence of Gottron's papules and heliotropic rash, $V$ area of the chest (shawl sign), myalgia of the upper limbs, and elevation of CK, LDH and transaminases in blood analyses, EMG, and histopathological findings. After the diagnosis of dermatomyositis, the patient was treated with regular oral prednisolone $(0.7 \mathrm{mg} / \mathrm{kg} /$ day $)$ with mediocre improvement. Due to reported dysphagia further tests were performed, which only revealed chronic gastritis with hyperplasia foveolaris mucosae. During subsequent hospitalization 6 months after the onset of symptoms, the patient was diagnosed with inoperable gastric cancer with liver metastases, and died 2 months later.

The factors predictive of malignancy in patients with dermatomyositis remain to be delineated. Male gender, more advanced age, absence of interstitial lung disease, and especially past history of diabetes mellitus have been hypothesized to play a vital role in the pathogenesis of this condition [5]. Various studies have suggested many different clinical and serological findings as possible predictive factors for the presence of malignancy in patients with dermatomyositis: age, increased erythrocyte sedimentation rate, presence of cutaneous leukocytoclastic vasculitis, cutaneous rash and skin lesions such as cutaneous necrosis and periungual erythema, neoplastic markers, or dysphagia $[4,6]$. Furthermore, an association between dermatomyositis, malignancy, and anti-TIF- $1 \gamma$ autoantibodies has been reported with $78 \%$ sensitivity for patients with dermatomyositis and concomitant cancer [7]. Both described patients were elderly and had severe skin lesions, but only one of them had dysphagia, antiTIF- $1 \gamma$ antibodies, and glucose disorders.

Although the factors causing dermatomyositis remain to be elucidated, in the aetiology co-occurrence of these two conditions it has been suspected that the malignant cells express certain cryptic antigens on their cell surface, generating autoantibodies that react with the muscle cells and cause myositis [8]. Moreover, it has been hypothesized that some viral and autoimmune factors may be involved, and that an immune response against cancer may trigger a reaction against the skeletal muscle [9].

In China lung, breast, gastric, colorectal, nasopharyngeal, and ovarian cancer have been the most common types of cancer among dermatomyositis patients in the last years [4]. After analysing data from Sweden, Denmark, and Finland, Hill et al. found that $32 \%$ of patients with dermatomyositis developed cancer, and that the most common types of malignancies were ovarian, lung, pancreatic, gastric and colorectal cancer, and non-Hodgkin lymphoma [2]. In the case of our patients, gastrointestinal (GI) neoplasms were diagnosed, similarly to a study using extensive testing for malignancy in dermatomyositis patients, which reported a $15 \%$ prevalence of GI malignancy $[10,11]$. Also, Cheng et al., in a large population-based study, reported a prevalence of GI malignancies as high as 5\% [12]. On the other hand, a cross-sectional study examining the results of upper $\mathrm{Gl}$ endoscopy and colonoscopy in adults with dermatomyosi- tis, with an observation period of more than 10 years, did not confirm a similar relationship, and identified no cases of GI cancer [12]. Due to these discrepancies, further studies are warranted to identify and understand the real risk of Gl cancer in patients with dermatomyositis.

The prognosis for dermatomyositis adult patients depends on the presence of concomitant cancer, and malignancies in dermatomyositis patients are associated with high mortality [5]. Remembering that dermatomyositis can be indicative of malignant neoplasms, diagnostics aimed at excluding accompanying proliferative processes should always be carried out. This applies especially to patients older than 40 years of age.

\section{Conflict of interest}

The authors declare no conflict of interest.

\section{References}

1. Stertz G. Polymyositis. Berl Klin Wochenschr 1916; 53: 489.

2. Hill CL, Zhang Y, Sigurgiersson B, et al. Frequency of specific cancer types in dermatomyositis and polymyositis: a populationbased study. Lancet 2001; 357: 96-100.

3. Fujita J, Tokuda M, Bandoh S, et al. Primary lung cancer associated with polymyositis/dermatomyositis, with a review of the literature. Rheumatol Int 2001; 20: 81-4.

4. Liu Y, Xu L, Wu H, et al. Characteristics and predictors of malignancy in dermatomyositis: analysis of 239 patients from northern China. Oncol Lett 2018; 16: 5960-8.

5. Motomura K, Yamashita H, Yamada S, et al. Clinical characteristics and prognosis of polymyositis and dermatomyositis associated with malignancy: a 25-year retrospective study. Rheumatol Int 2019; 39: 1733-9.

6. Di Rollo D, Abeni D, Tracanna M, et al. Cancer risk in dermatomyositis: a systematic review of the literature. Ital Dermatol Venereol 2014; 149: 525-37.

7. Fiorentino DF, Kuo K, Chung L, et al. Distinctive cutaneous and systemic features associated with antitranscriptional intermediary factor- $1 \gamma$ antibodies in adults with dermatomyositis. J Am Acad Dermatol 2015; 72: 449-55.

8. Zhang W, Jiang SP, Huang L Dermatomyositis and malignancy: a retrospective study of 115 cases. Eur Rev Med Pharmacol Sci 2009; 13: 77-80.

9. Kao LL, Chung L, Fiorentino DF. Pathogenesis of dermatomyositis: role of cytokines and interferon. Curr Rheumatol Rep 2011; 13: 225-32.

10. Sparsa A, Liozon E, Herrmann F, et al. Routine vs extensive malignancy search for adult dermatomyositis and polymyositis: a study of 40 patients. Arch Dermatol 2002; 138: 885-90.

11. Kidambi TD, Schmajuk G, Gross AJ, et al. Endoscopy is of low yield in the identification of gastrointestinal neoplasia in patients with dermatomyositis: a cross-sectional study. World J Gastroenterol 2017; 23: 4788-95.

12. Chen YJ, Wu CY, Huang YL, et al. Cancer risks of dermatomyositis and polymyositis: a nationwide cohort study in Taiwan. Arthritis Res Ther 2010; 12: R70. 